\title{
Shifting from implicit to explicit knowledge: Different roles of early- and late-night sleep
}

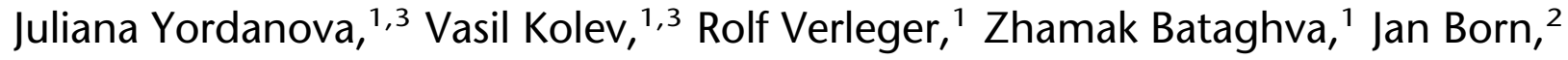 \\ and Ullrich Wagner ${ }^{1,2,4,5}$ \\ ${ }^{1}$ Department of Neurology, University of Lübeck, 23538 Lübeck, Germany; ${ }^{2}$ Department of Neuroendocrinology, University \\ of Lübeck, 23538 Lübeck, Germany; ${ }^{3}$ Institute of Neurobiology, Bulgarian Academy of Sciences, 1113 Sofia, Bulgaria; \\ ${ }^{4}$ Department of Fundamental Neuroscience, University Medical Center, 1211 Geneva, Switzerland
}

\begin{abstract}
Sleep has been shown to promote the generation of explicit knowledge as indicated by the gain of insight into previously unrecognized task regularities. Here, we explored whether this generation of explicit knowledge depends on pre-sleep implicit knowledge, and specified the differential roles of slow-wave sleep (SWS) vs. rapid eye movement (REM) sleep in this process. Implicit and explicit knowledge (insight) related to a hidden regularity were assessed in an associative motor-learning task (number reduction task, NRT), which was performed in two sessions (initial practice and retest) separated by $3 \mathrm{~h}$ of either early-night sleep, rich in SWS, or of late-night sleep, rich in REM sleep. About half of the participants developed signs of implicit rule knowledge (i.e., speeded reaction times for responses determined by the hidden regularity) at initial practice preceding early or late sleep. Of these, half developed explicit knowledge across early-night sleep, significantly more than across late-night sleep. In contrast, late-night subjects preferentially remained on the level of implicit rule knowledge after sleep. Participants who did not develop implicit knowledge before sleep had comparable rates of transition to implicit or explicit knowledge across early and late sleep. If subjects gained explicit knowledge across sleep, this was associated with lower amounts of REM sleep, specifically in the late-night group. SWS predominant during the early night may restructure implicit memory representations in a way that allows creating an explicit representation afterward, whereas REM sleep in the late night appears to stabilize them in their implicit form.
\end{abstract}

Two types of knowledge, explicit and implicit, have been phenomenologically distinguished in humans (Reber 1989; Seger 1994; Dienes and Perner 1999). Explicit knowledge is acquired through attentive and intentional monitoring of external and internal events and can be used for deliberate control of behavior. Implicit knowledge is acquired unintentionally, with subjects being not or little aware of its presence. At the neurobiological level, a distinction has been made between a hippocampus-dependent memory system subserving explicit memory formation, and a more heterogeneous hippocampusindependent system underlying different types of implicit memory formation (Squire 1992; Reber and Squire 1994; Forkstam and Petersson 2005).

Sleep has been identified as a critical brain state involved in both explicit and implicit memory consolidation (for overviews, see Maquet 2001; Smith 2001; Paller and Voss 2004; Born et al. 2006; Walker and Stickgold 2006), where consolidation refers to a post-learning process that stabilizes and strengthens the new memory traces established at learning (Lechner et al. 1999; McGaugh 2000). Recent studies have shown that in this process sleep not only stabilizes but also reorganizes memory representations such that performance after sleep can qualitatively differ from what has been learned originally (Fenn et al. 2003; Wagner et al. 2004; Gómez et al. 2006; Ellenbogen et al. 2007). This cognitive reorganization has become particularly salient in one of these studies by a dramatic change in overt task performance across sleep (Wagner et al. 2004). Subjects performed the socalled number reduction task (NRT), where in each trial a sequence of digits has to be transformed into a new sequence according to predefined rules, with the last digit of the new sequence defined as

${ }^{5}$ Corresponding author.

E-mail wagner@kfg.uni-luebeck.de; fax 49-451-5003640.

Article is online at http://www.learnmem.org/cgi/doi/10.1101//m.897908. the "final result" to be determined (Woltz et al. 1996; Frensch et al. 2002; Haider and Rose 2007). Importantly, a hidden regularity was implemented in all task trials. Acquiring explicit knowledge of this hidden regularity (i.e., gaining insight into it) allows subjects to abruptly shortcut processing of the sequences (Fig. 1A). The NRT thus represents both an implicit motor-learning task, in which learning progress can be observed as a gradual speeding of sequential stimulus-response processing, and an associative learning task, in which abstract associations can or cannot become accessible to awareness. The key finding of Wagner et al. (2004) was that sleep strongly enhanced the probability of gaining insight into the covert task structure. This was evidenced by a substantially higher number of subjects who discovered the hidden regularity when the task was performed after periods of sleep compared with wakefulness of equal duration, with both sleep and wake periods preceded by practice on the task serving to create an initial task representation in memory. Thus, sleep after learning supported a process that subsequently facilitated explicit knowledge generation, resulting in qualitatively improved task performance. The aim of the present study was to investigate the sleep-related mechanisms of this process in more detail.

Two major questions were addressed. The first was whether post-sleep explicit knowledge (insight) represents genuinely new knowledge or emerges basically from the transformation of implicit knowledge of the hidden regularity already acquired before sleep. Accordingly, NRT performance after sleep was evaluated in relation to whether or not subjects had gained implicit knowledge already before sleep. In the NRT, the acquisition of implicit associative knowledge specifically related to the hidden task structure is indicated by a relative speeding of responses to digits that are determined by the hidden structure compared with those that are not determined (for details, see Materials and Methods) (Frensch et al. 2002; Rose et al. 2002, 2004, 2005; Lang et al. 2006). Given previous findings that consolidation effects of 
A

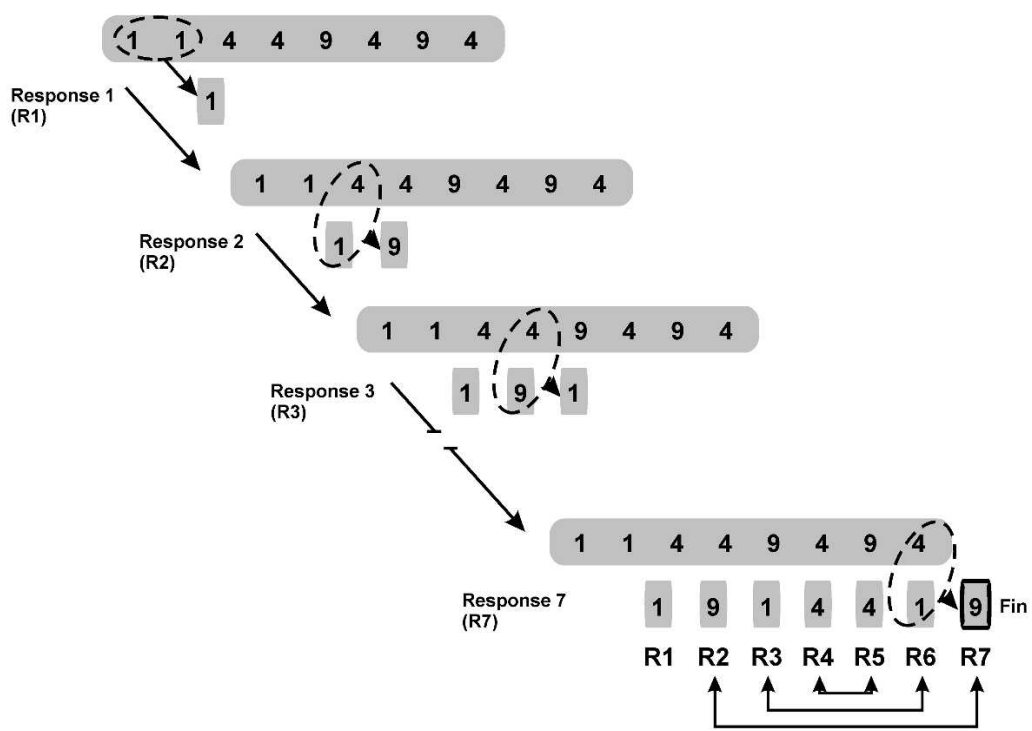

B

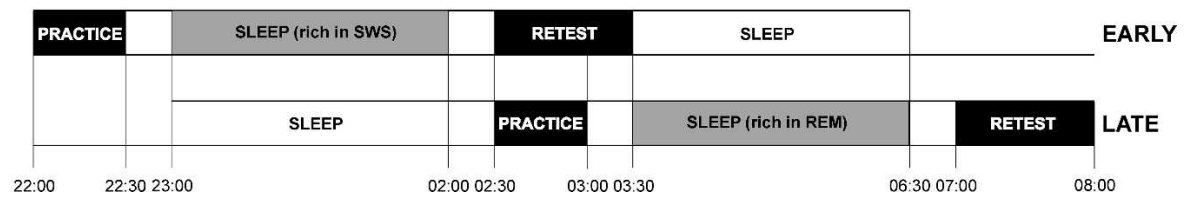

Figure 1. Experimental design. $(A)$ Number reduction task (NRT), illustrated by an example trial. Subjects sequentially transform a given sequence of digits (with only the three digits 1, 4, and 9 used) into a new sequence to determine a specific digit as the final result to this trial (Fin). This could be achieved by sequentially processing pairs of digits from left to right according to two simple rules, i.e., the "identity rule" (stating that the result of two identical digits is the same digit, e.g., 1 and 1 gives 1 , as in Response 1 here) and the "difference rule" (stating that the result of two nonidentical digits is the remaining third digit of this three-digit system, e.g., 1 and 4 gives 9, as in Response 2 here). The result digit of a pairwise transformation always serves as one of the two digits to be transformed in the next step. This sequential processing thus results in the creation of a sequence of seven response digits (R1-R7). The hidden task structure implemented in all task trials is that the last three response digits are mirroring the previous three response digits (illustrated by pairwise arrows here), which implies that the second response digit always equals the final result $(\mathrm{R} 2=\mathrm{R} 7)$. Gaining explicit knowledge (insight) of this rule allows an early determination of the final result already after the second response. For more details, see Materials and Methods. (B) Experimental protocol. NRT sessions of initial practice and retesting are marked for the two experimental groups. The critical interval between initial practice and retest contained particularly high amounts of either SWS (early-night group) or REM sleep (late-night group).

sleep can depend on the level and type of task knowledge achieved at learning (Peigneux et al. 2003; Kuriyama et al. 2004; Robertson et al. 2004; Hauptmann et al. 2005; Peters et al. 2007), it was expected that gaining insight after sleep would depend on whether implicit knowledge about the regularity was indeed acquired before sleep.

The second question was how different sleep stages, specifically slow-wave sleep (SWS) and rapid eye movement (REM) sleep, are involved in sleep-associated generation of explicit knowledge of the hidden structure. SWS has been implicated in the consolidation of hippocampus-dependent explicit (or declarative) memory tasks, while REM sleep seems to be particularly pertinent to hippocampus-independent implicit (or nondeclarative) tasks (Plihal and Born 1997, 1999; Peigneux et al. 2003, 2004; Wagner et al. 2003, 2007; Marshall et al. 2007). However, little is known about the role of these two sleep stages in tasks like the NRT that involve both implicit and explicit aspects and, more specifically, in which explicit knowledge may emerge from previous implicit processing. To separate the effects of SWS vs. REM sleep, changes in task performance were assessed across sleep periods in the early half of the night, containing high amounts of SWS, and across sleep periods in the late half of the night, containing high amounts of REM sleep (Fig. 1B). This procedure of night-half comparison has been efficiently applied in several previous studies, which revealed differential effects of SWS-rich and REM sleeprich periods (e.g., Fowler et al. 1973; Plihal and Born 1997, 1999; Wagner et al. 2001, 2003). These and other studies (Peigneux et al. 2003, 2004; Rasch et al. 2007) have pointed to critical roles of SWS for explicit memories and of REM sleep for implicit memories. It was therefore expected that more subjects would generate explicit knowledge of the hidden regularity across SWS-rich early sleep than across REM sleep-rich late sleep.

\section{Results}

The same version of the NRT was used as in Wagner et al. (2004), with the critical hidden regularity being the mirror structure of response strings generated in each task trial (for details, see Fig. 1A; Materials and Methods). Subjects performed on two task sessions, a pre-sleep session, serving as an initial practice session to build up a first mental representation of the task (three blocks), and a post-sleep retest session (10 blocks). However, unlike in the previous study, the critical interval between the two sessions was not filled with whole-night sleep, but either with $3 \mathrm{~h}$ of early-night sleep, rich in SWS (early-night group), or with $3 \mathrm{~h}$ of late-night sleep, rich in REM sleep (late-night group) (Fig. 1B). Sleep recordings confirmed the differential distribution of SWS vs. REM sleep (Table 1). Subjects in the early-night group had substantially more SWS than did those in the late-night group $(P<0.001)$, and subjects in the late-night group, conversely, had substantially more REM sleep than did those in the early-night group $(P<0.001)$. The two groups did not differ in the proportions of other sleep stages $(P>0.15)$.

\section{Development of implicit and explicit knowledge related to the hidden regularity}

Three types of knowledge states were defined with regard to the hidden regularity: (1) subjects could have no knowledge (NoK) about the hidden structure, which was of course the initial knowledge state for all subjects; (2) they could have implicit knowledge (ImK), as indicated by faster reaction times (RTs) for responses that were fully determined by the hidden task structure (see below, for details); or (3) they could have developed explicit knowledge (ExK) by gaining insight related to the hidden structure, the final level of knowledge allowing immediate shortcutting of performance on the sequences. Subjects who gained explicit knowledge already at pre-sleep practice (early-night group: $n=4$; late- 
Table 1. Distribution of sleep stages in the early- vs. late-night group

\begin{tabular}{lcrrr}
\hline & $\begin{array}{c}\text { Early-night } \\
\text { group }\end{array}$ & \multicolumn{1}{c}{$\begin{array}{c}\text { Late-night } \\
\text { group }\end{array}$} & \multicolumn{2}{c}{ Early vs. late } \\
\cline { 4 - 5 } & \multicolumn{1}{c}{$\boldsymbol{F}_{(1,54)}$} & $\boldsymbol{P}$ \\
\hline Wake (\%) & $3.06 \pm 1.16$ & $0.44 \pm 1.41$ & 2.07 & 0.16 \\
S1 (\%) & $7.60 \pm 1.0$ & $7.80 \pm 1.20$ & 0.02 & 0.88 \\
S2 (\%) & $57.80 \pm 2.27$ & $61.38 \pm 2.76$ & 1.01 & 0.39 \\
SWS (\%) & $25.69 \pm 2.20$ & $9.54 \pm 2.67$ & 21.72 & $<\mathbf{0 . 0 0 1}$ \\
REM (\%) & $5.62 \pm 0.96$ & $20.72 \pm 1.17$ & 98.92 & $<\mathbf{0 . 0 0 1}$ \\
Total sleep & & & & \\
$\quad$ time (min) & $190.0 \pm 3.69$ & $191.8 \pm 4.49$ & 0.09 & 0.77 \\
\hline
\end{tabular}

S1 indicates sleep stage 1; S2, sleep stage 2; SWS, slow-wave sleep; and REM, rapid eye movement sleep. Means \pm SEM are indicated. Data refer to the sleep interval between initial practice and retesting. Statistical results are from one-way ANOVA comparing early- and late-night groups. Significant $P$-values are in bold.

night group: $n=5$ ) were excluded, because subsequent sleep could not have any further effect in this case.

To evaluate differences between early and late sleep with respect to the change of knowledge states across sleep, the nighthalf groups were first subdivided according to the knowledge state acquired at initial practice before sleep (either NoK or ImK). About half of the subjects acquired ImK already during initial practice (total: 47.3\%; early-night group: 44.8\%, late-night group: 50.0\%), while the other subjects remained on the NoK level before sleep. The subgroups of subjects with pre-sleep NoK and pre-sleep ImK were then further analyzed by which state of knowledge the subjects achieved after sleep (NoK, ImK, or ExK). This resulted in six subgroups for each night-half (Table 2; Fig. 2). Separately for the two performance states before sleep (pre-ImK/ pre-NoK), transitions to knowledge states after sleep (post-NoK, post-ImK, and post-ExK) were compared between early-night and late-night sleep conditions.

For subjects who had acquired implicit knowledge already before sleep (pre-ImK), as illustrated in Figure 2, it depended on the sleep condition whether their implicit knowledge before sleep was maintained or transformed after sleep $\left(\chi^{2}(2)=6.16\right.$, $P=0.046) ;$ the same statistical outcome also held when the small group of subjects who fell back to the NoK level after sleep was disregarded in this analysis $\left(\chi^{2}(1)=5.79, P=.016\right)$. Pairwise specifications of this pattern showed that early sleep substantially increased the probability of shifting to explicit knowledge after sleep compared with late sleep $(85.7 \%$ vs. $14.3 \%, P=0.004$, in pre-ImK to post-ExK group), while late compared with early sleep was associated with a higher probability of maintaining the level of implicit knowledge after sleep $(68.8 \%$ vs. $31.2 \%, P=0.05$, in pre-ImK to post-ImK group).

Unlike the pre-ImK subjects, individuals who had remained at the NoK level before sleep (pre-NoK) were equally likely to attain NoK, ImK, or ExK after sleep (Fig. 2). The rates of transformation of NoK state of knowledge across sleep did not differ between early- and late-night groups $\left(\chi^{2}(2)=1.88, P=0.4\right)$. However, having gained $\operatorname{ImK}$ before sleep was not a general precondition for gaining explicit knowledge afterward: Across night-half conditions, subjects who achieved explicit knowledge after sleep were equally likely to have been on the level of ImK or NoK at initial practice $(26.9 \%$ vs. $\left.24.1 \% ; \chi^{2}(1)=0.06, P=0.8\right)$.

In a separate analysis, the acquired states of knowledge after sleep were also compared between the two night-half groups regardless of pre-sleep knowledge states. The proportion of ImK subjects was higher after late- than early-night sleep $\left(\chi^{2}(1)=5.15\right.$, $P=0.02)$, whereas the proportion of ExK subjects after sleep did not overall differ between the two night-half groups $\left(\chi^{2}(1)=1.01\right.$, $P=0.32$ ).

\section{Sleep stages associated with knowledge states}

Within early- and late-night groups, the distribution of sleep stages was compared between the subgroups formed according to the knowledge state after sleep (Table 3 ). There were no differences between subgroups of the early-night group. However, in the late-night group, subjects who gained explicit knowledge after sleep had substantially less REM sleep than ImK and NoK subjects (post-ExK: $13.50 \%$, post-ImK: $23.71 \%$, post-NoK: $24.94 \% ; P=0.002$ ) (Table 3). Subsamples were too small to perform more fine-grained statistical analyses on sleep also for the different pre-/post-knowledge state combinations. However, like for post-sleep knowledge states regardless of pre-sleep states, distributions of sleep stages were also compared between the two pre-sleep knowledge states (pre-ImK vs. pre-NoK) regardless of post-sleep states, revealing that in the late-night group subjects with pre-sleep ImK showed larger amounts of REM sleep and less S2 sleep than subjects without pre-sleep ImK (REM sleep: $24.75 \%$ vs. $19.2 \% ; F_{(1,25)}=5.19, P<0.05$; S2: $54.7 \%$ vs. $65.2 \%$; $\left.F_{(1,25)}=9.89, P<0.005\right)$. In the same analysis performed for the early-night group, none of the sleep parameters differed between subjects with and without markers of $\operatorname{ImK}$ before sleep $(P>0.3)$.

\section{Reaction time (RT) analyses}

RT analyses were performed to validate the distinction between ImK and NoK subjects on the group level. According to our classification (for details, see Materials and Methods), ImK subjects, but not NoK subjects, should display distinctively faster response times for responses R5-R7 that were predictable due to the mirror structure in the response strings than to unpredictable responses R2-R4 (Fig. 1A). The RT analyses indeed confirmed this behavioral difference, both in pre-sleep and in post-sleep classifications. For pre-sleep comparisons, an ANOVA with the between-subjects
Table 2. Distribution of subjects according to their state of knowledge before and after sleep

(A) Pre-NoK

\begin{tabular}{|c|c|c|c|c|c|}
\hline & Total & Pre-Nok & $\begin{array}{l}\text { Pre-NoK } \\
\downarrow \\
\text { Post-NoK }\end{array}$ & $\begin{array}{l}\text { Pre-NoK } \\
\downarrow \\
\text { Post-ImK }\end{array}$ & $\begin{array}{l}\text { Pre-NoK } \\
\downarrow \\
\text { Post-ExK }\end{array}$ \\
\hline Early-night group & 29 & $16(55.2 \%)$ & $9(56.3 \%)$ & $4(25.0 \%)$ & $3(18.7 \%)$ \\
\hline Late-night group & 26 & $13(50.0 \%)$ & $4(30.8 \%)$ & $5(38.5 \%)$ & $4(30.7 \%)$ \\
\hline
\end{tabular}

(B) Pre-ImK

\begin{tabular}{|c|c|c|c|c|c|}
\hline & Total & Pre-ImK & $\begin{array}{l}\text { Pre-ImK } \\
\downarrow \\
\text { Post-NoK }\end{array}$ & $\begin{array}{l}\text { Pre-ImK } \\
\downarrow \\
\text { Post-ImK }\end{array}$ & $\begin{array}{l}\text { Pre-ImK } \\
\downarrow \\
\text { Post-ExK }\end{array}$ \\
\hline Early-night group & 29 & $13(44.8 \%)$ & $2(15.4 \%)$ & $5(38.4 \%)$ & $6(46.2 \%)$ \\
\hline Late-night group & 26 & $13(50.0 \%)$ & $1(7.7 \%)$ & $11(84.6 \%)$ & $1(7.7 \%)$ \\
\hline
\end{tabular}

Indicated are numbers (rates) of subjects from the early- and late-night groups displaying different post-sleep states of knowledge about the hidden task structure at retesting, depending on whether they had no knowledge (A) or implicit knowledge (B) already at initial practice before sleep. Pre-NoK indicates pre-sleep no knowledge; pre-ImK, pre-sleep implicit knowledge; post-NoK, post-sleep no knowledge; post-ImK, post-sleep implicit knowledge; post-ExK, post-sleep explicit knowledge; and $\downarrow$, transition of knowledge state from pre- to post-sleep. 


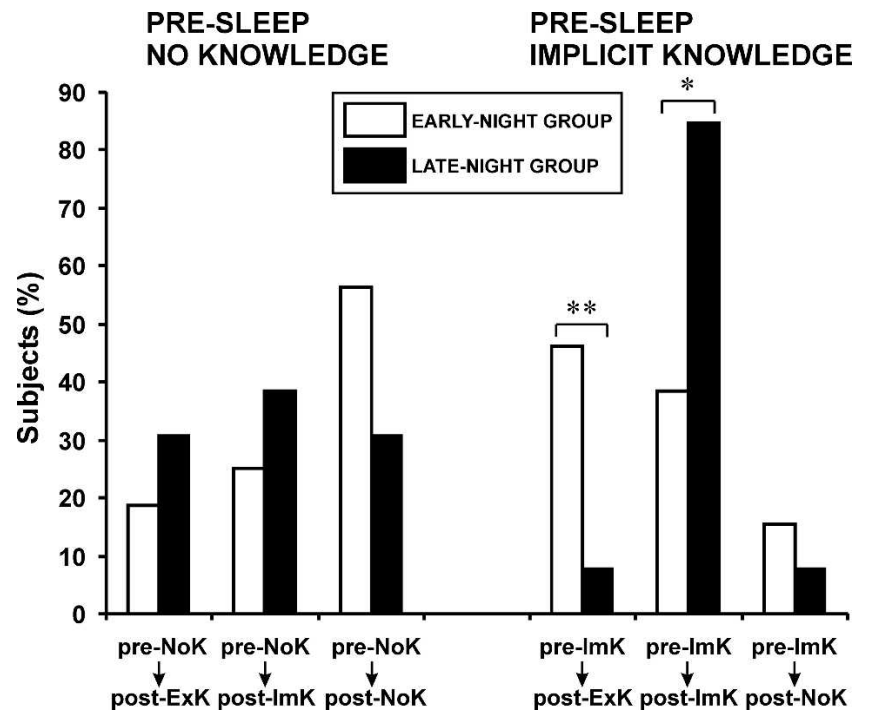

Figure 2. Percentages of subjects who preserved/transformed their state of knowledge (as presented in Table 2 ) across early and late sleep. ${ }^{*} P=0.05 ;{ }^{* *} P<0.01$.

factors knowledge state (ImK/NoK) and sleep condition (early/ late), and the within-subjects factors predictability (R2-4/R5-7) and block (1-3) revealed that predictable responses were generally faster than unpredictable ones (main effect of predictability, $\left.F_{(1,51)}=128.52, P<0.0001\right)$, and that this was particularly due to a substantial speeding of predictable relative to unpredictable responses in ImK but not in NoK subjects (knowledge state $\times$ predictability interaction; $\left.F_{(1,51)}=13.38, P<0.0001\right)$ (Fig. 3A). No other main effect or interaction was significant. The same pattern of effects was obtained for post-sleep comparisons $(10$ blocks of retesting: main effect of predictability, $F_{(1,37)}=75.73$, $P<0.0001$; knowledge state $\times$ predictability interaction, $\left.F_{(1,37)}=6.34, P=0.015\right)$, with the only additional significant finding that ImK subjects also responded generally faster than NoK subjects (main effect of knowledge state, $F_{(1,37)}=5.82$, $P=0.021$ ) (Fig. 3B).

In an analogous ANOVA, we also compared subjects who developed ExK (insight) after sleep with those who did not, with respect to off-line progress in implicit learning across sleep, as reflected in RT changes from the last block of initial training to the first block of retesting. All subjects became faster from the last block of initial training to the first block of retesting (main effect of block, $\left.F_{(1,51)}=28.5, P<0.0001\right)$, and this general speeding did not depend on whether subjects later developed ExK, or whether the sleep period took place in the early or late night $(P>0.40$, for block $\times$ knowledge state and block $\times$ sleep condition interactions). There was only a statistical trend in the direction that the decrease in response times across sleep was somewhat less pronounced in late-night subjects who subsequently gained insight than in other subjects $(P<0.10$, for the interaction of block $\times$ knowledge state $\times$ sleep condition). The extent of speeding and late sleep across sleep did not differ between predictable vs. unpredictable responses ( $P>0.60$, for all respective ANOVA interactions).

\section{Subjective ratings}

Ratings of subjective feelings of sleepiness, activation, tension, boredom, motivation, and concentration were obtained before and after each session of initial practice and retest. The two sleep conditions did not differ on the whole in these variables, as indicated by nonsignificant main effects of early vs. late night (all $P>0.17)$. However, subjects felt somewhat more sleepy and less activated, motivated, and concentrated in task sessions performed after $3 \mathrm{~h}$ of sleep in the middle of the night (i.e., initial practice for late-night group, retest for early-night group) than in sessions performed in the evening (initial practice for early-night group) or in the morning (retest for late-night group) $(P<0.05$, for respective night-half $\times$ session interactions). A much stronger effect independent of sleep was an activating effect of task performance itself; i.e., subjects felt less sleepy and more activated at the end compared with the beginning of a task session $(P<0.001)$.

\section{Discussion}

This study specified the role of sleep in promoting insight into a hidden abstract regularity in the NRT (Wagner et al. 2004). The basic aim was to elucidate the contribution of pre-sleep implicit knowledge as well as the role of different sleep stages (SWS vs. REM sleep) in the generation of insight, i.e., explicit knowledge, across sleep. The central finding was that when subjects had already developed signs of implicit knowledge of the hidden regularity at initial training before sleep, the proportion of subjects gaining explicit knowledge of the regularity was substantially higher after early sleep, whereas after late sleep most of these subjects remained on the level of implicit knowledge. This result points to a critical role of SWS, predominantly occurring in the early night, for transforming implicit to explicit knowledge. In this process, the cortico-hippocampal system may be a key mediator (McClelland et al. 1995). Neuroimaging studies have shown that accumulation of implicit knowledge during the NRT as used here is accompanied by activation of the hippocampal memory system in the medial temporal lobe (Rose et al. 2002, 2004, 2005).

Table 3. Distribution of sleep stages in subjects with different state of knowledge after early

\begin{tabular}{lrrrrrr}
\hline & \multicolumn{2}{c}{ State of knowledge achieved at retesting } & & \\
\cline { 2 - 4 } $\begin{array}{l}\text { Sleep } \\
\text { parameter }\end{array}$ & \multicolumn{1}{c}{$\begin{array}{c}\text { Explicit } \\
\text { (ExK) }\end{array}$} & \multicolumn{1}{c}{$\begin{array}{c}\text { Implicit } \\
\text { (ImK) }\end{array}$} & $\begin{array}{c}\text { No knowledge } \\
\text { (NoK) }\end{array}$ & & \\
\hline Early sleep & & & & & \\
$\quad$ Wake (\%) & $6.28 \pm 2.07$ & $2.62 \pm 2.07$ & $0.29 \pm 1.87$ & 1.25 & 0.30 \\
S1 (\%) & $8.55 \pm 1.79$ & $8.00 \pm 1.80$ & $6.25 \pm 1.62$ & 0.38 & 0.70 \\
S2 (\%) & $55.89 \pm 4.06$ & $56.90 \pm 4.06$ & $60.55 \pm 3.68$ & 0.32 & 0.80 \\
SWS (\%) & $24.90 \pm 3.94$ & $25.83 \pm 3.94$ & $26.36 \pm 3.56$ & 0.03 & 0.97 \\
REM (\%) & $4.09 \pm 1.72$ & $6.43 \pm 1.72$ & $6.34 \pm 1.56$ & 0.63 & 0.54 \\
Total sleep time (min) & $181 \pm 6.61$ & $192 \pm 6.60$ & $197 \pm 5.98$ & 1.40 & 0.27 \\
Late sleep & & & & & \\
Wake (\%) & $0.40 \pm 2.77$ & $0.46 \pm 1.55$ & $0.46 \pm 2.77$ & 0.003 & 1.00 \\
S1 (\%) & $9.32 \pm 2.41$ & $6.45 \pm 1.35$ & $7.72 \pm 2.41$ & 0.90 & 0.40 \\
S2 (\%) & $64.30 \pm 5.45$ & $58.01 \pm 3.05$ & $61.84 \pm 5.45$ & 0.86 & 0.40 \\
SWS (\%) & $12.66 \pm 5.28$ & $11.22 \pm 2.95$ & $4.74 \pm 5.28$ & 1.16 & 0.30 \\
REM (\%) & $13.50 \pm 2.31$ & $23.71 \pm 1.29$ & $24.94 \pm 2.31$ & 8.06 & $\mathbf{0 . 0 0 2}$ \\
$\quad$ Total sleep time (min) & $184 \pm 8.86$ & $191 \pm 4.96$ & $201 \pm 8.86$ & 1.05 & 0.36 \\
\hline
\end{tabular}

S1 indicates sleep stage 1; S2, sleep stage 2; SWS, slow-wave sleep; and REM, rapid eye movement sleep. Means \pm SEM are indicated. For each of the two experimental groups (early-/late-night group), data from Table 1 are analyzed by subgroups of subjects showing explicit (ExK), implicit (ImK), or no knowledge (NoK) of the hidden task structure at retesting after sleep. Statistical results are from one-way ANOVA comparing the three knowledge states. Significant $P$-values are in bold.

aEarly sleep, $F_{(2,28)}$; late sleep, $F_{(2,25)}$. 
A

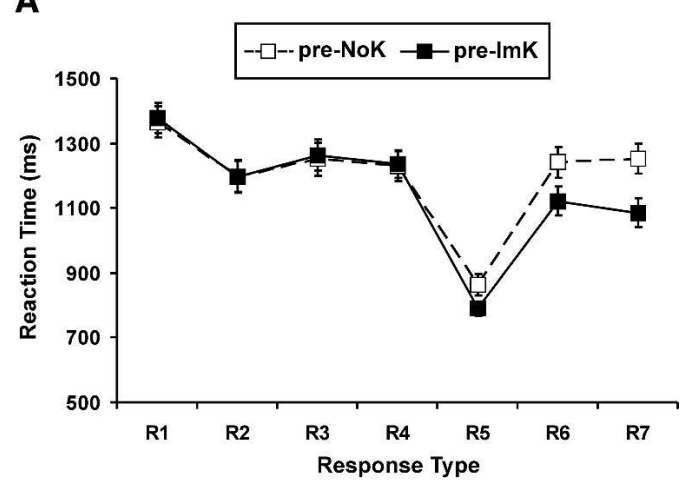

B

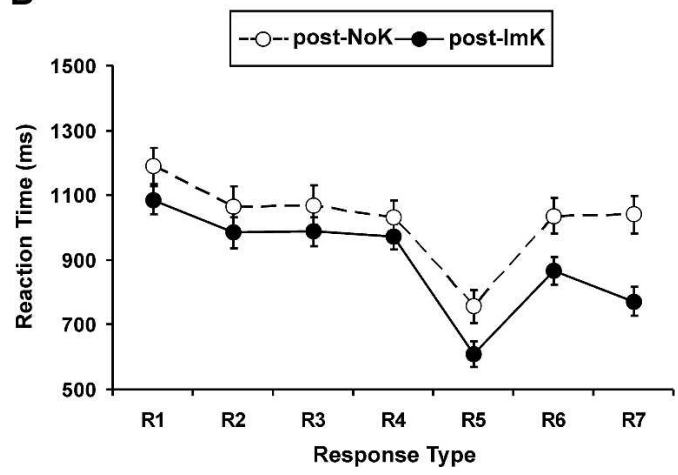

Figure 3. Reaction times (mean $\pm \mathrm{SEM}$ ) for the seven different responses (R1-R7) generated in each trial of the NRT for subjects classified as having developed implicit knowledge of the hidden structure (ImK) or not (NoK) at initial practice before sleep (pre-NoK vs. pre-ImK, panel $A$, averaged across the three task blocks) and at retesting after sleep (postNoK vs. post-ImK, panel $B$, averaged across the 10 task blocks). ImK subjects show faster reaction times than NoK subjects particularly for responses R5-R7 that are determined due to the mirror structure (as shown in Fig. $1 \mathrm{~A}$ ) in relation to the undetermined responses R2-R4. Reaction times for R5 are in all subjects distinctly shorter than for all other responses because it is always a direct repetition of R4. For details, see Materials and Methods.

Also, the hippocampus has been found to be involved in processes of assessing relationships between events and putting events into their context (Cohen et al. 1999; Henke et al. 1999; Elsner et al. 2002; Bird and Burgess 2008). SWS has been specifically implicated in the consolidation of hippocampus-dependent memories, presumably as a result of reactivation of hippocampal neurons and associated hippocampo-neocortical information transfer during this sleep stage (Wilson and McNaughton 1994; Buzsáki 1998; Gais and Born 2004; Wagner and Born 2008). Hence, hippocampal activation associated with the gain of implicit associative knowledge before sleep may be continued by reactivation during SWS, which would substantiate the transfer of implicit information to the neocortex and may explain the selective effect of only early-night sleep on shifting from implicit to explicit knowledge.

REM sleep, in contrast, even seems to counteract the process of turning implicit knowledge into an explicit representation. This is suggested by two current pertinent observations. First, subjects who gained explicit knowledge across sleep had less REM sleep than those who did not. This was only significant for the second half of the night, possibly due to the low overall amount of REM sleep in the first half. Second, overall more subjects manifested implicit knowledge after late-night sleep, rich in REM sleep, than after early-night sleep, rich in SWS. This pattern is consistent with previous findings of a critical role for REM sleep in implicit memory formation (Plihal and Born 1997, 1999; Peigneux et al. 2003; Wagner et al. 2003) but also entails new implications. Because most subjects had acquired implicit knowledge already before the sleep period, the prevalence of implicit knowledge after late-night sleep suggests that REM sleep in the late night primarily stabilizes and preserves implicit representations rather than generating them. At the same time, as indexed by the low rate of transformation of implicit into explicit knowledge after late-night sleep, this stabilization of implicit knowledge by REM sleep may prevent a restructuring of the memory trace that is necessary to turn it into an explicit representation. It seems that the brain, when facing an initial implicit knowledge representation in the NRT, has two alternatives of processing it further during sleep, either stabilizing it (the way of processing associated with REM sleep in the late night) or actively restructuring it (the way of processing associated with SWS in the early night). The restructuring mode of processing may be the favored one under natural circumstances because early-night sleep inherently precedes late-night sleep. However, it is also possible that during a regular full night of sleep combined and more complex interactive effects of SWS in the early night and REM sleep in the late night play a role, which were not observable here due to the specific experimental design that intentionally separated the effects of early- and late-night sleep. Theoretically, REM sleep in the late night could additionally transform some of the restructuring effects that occurred during SWS in the preceding early part of the night.

Since early- and late-nocturnal sleep inherently take place at different times of the day, circadian factors modifying cognitive functioning independent of sleep might have influenced the results. Some variables of subjective feelings indeed differed according to the time points of testing, indicating more sleepiness and less activation and concentration in the middle of the night compared with the evening and the morning, although these circadian influences were relatively small compared to activating effects of task performance per se. Thus, retesting in the earlynight group and initial practice in the late-night group may have been influenced by somewhat reduced cognitive functioning in the middle of the night. However, the pattern of results speaks against a substantial impact of these factors: At initial practice, nearly the same proportion of subjects developed implicit knowledge in the early- and late-night groups, despite the different time-points of practice. Of most importance, even more of these subjects gained explicit insight into the hidden task structure at retest in the early-night than the late-night group, although the retest session in the early-night but not in the late-night group took place at the less favorable time-point in the middle of the night. Higher cognitive functions of creativity and divergent thinking are known to be the most impaired cognitive capabilities under conditions of strong sleepiness (Horne 1988; Durmer and Dinges 2005). Thus, if sleepiness were a critical factor here, an even lower proportion of subjects gaining insight would have been expected after early- than late-night sleep (although we cannot exclude the theoretical possibility of an influence of circadian factors in an interactive form, i.e., low alertness at retesting in combination with higher alertness at initial practice, as observed in the early night condition, could be more beneficial for gaining insight than the opposite pattern of higher alertness at retesting in combination with low alertness at initial practice, as observed in the late night condition).

The essential finding that generation of explicit knowledge was only enhanced after early sleep if implicit knowledge was already acquired before, adds to previous findings of sleepassociated interactions between explicit and implicit knowledge generation in the serial reaction time task (SRTT) (e.g., Fischer et al. 2006; Brown and Robertson 2007). For example, Fischer et al. 
(2006) found that subjects who had developed implicit knowledge of the repeated structure in a training session of the SRTT were able to correctly predict elements of the structure in a test of explicit knowledge after a post-training period of sleep compared with wakefulness. However, the differential role of different sleep stages was not addressed in their study, and because all subjects had developed implicit knowledge before sleep, the effect that sleep would have in subjects without pre-sleep implicit knowledge in this task remained unknown.

The NRT used here also has the methodological advantage that the emergence of explicit knowledge can be detected as a punctual event of insight, whose time point can be reliably determined (for a detailed comparison between NRT and SRTT, see Haider and Rose 2007). However, it could be argued also in the NRT that the transition from no knowledge to implicit knowledge represents a dynamic continuum rather than a clear-cut change of knowledge states. Then, subjects with implicit knowledge should be regarded only as more advanced in the learning process than subjects without implicit knowledge. Even so, however, the present results would still indicate that the differential effects of early and late sleep depend on the level of task performance that was already achieved before sleep (e.g., Peigneux et al. 2003; Hauptmann et al. 2005; Peters et al. 2007).

It is to be noted that the type or level of pre-sleep knowledge as defined here is a factor that clearly influences, but not absolutely determines, whether explicit knowledge can be developed across subsequent sleep. In fact, half of the overall 14 subjects who gained explicit insight into the hidden rule after sleep had not developed implicit rule knowledge before sleep. Thus, insight can be gained also by processes independent of pre-sleep performance that are equally affected by early and late sleep. Partly, these processes are even independent of sleep at all, because a certain number of subjects also gain insight in the NRT without sleep (Rose et al. 2002; Wagner et al. 2004; Lang et al. 2006). The contribution of such additional factors influencing insight cannot be evaluated on the basis of the present data. However, the critical point demonstrated here is that those factors that do depend on sleep are clearly differentially affected by early and late sleep in relation to the level of pre-sleep knowledge, suggesting a specific role for SWS in turning pre-sleep implicit knowledge into explicit knowledge, and a specific role for REM sleep in preserving previously acquired implicit knowledge after sleep.

In sum, after pre-sleep activation of the implicit memory system, SWS-rich early-night sleep has the ability to facilitate subsequent generation of explicit knowledge of previously uncomprehended abstract information. In contrast, REM sleeprich-late sleep in the first place appears to stabilize implicit representations without changing their structure. Thus, only earlynight sleep, presumably by way of its high SWS proportion, is specifically associated with a reorganization of implicit memory representations that promotes accessibility to awareness after sleep.

\section{Materials and Methods}

\section{Subjects}

Sixty-eight healthy students (18-28 yr old) without any history of sleep disturbances or psychiatric or neurological disorders participated in the experiments. All subjects spent an adaptation night in the sleep laboratory, including placement of electrodes. Subjects were paid for their participation and gave informed written consent prior to the study, which was approved by the local ethics committee. Participants who gained explicit knowledge already at initial practice (indicated by shortcut responding in the NRT) were excluded from further analyses (four subjects in the early-night group and five subjects in the late-night group).
One more subject was excluded from the early-night group because of staying awake $40 \%$ of the time, and one subject from the early-night group and two more subjects from the late-night group were excluded because of low quality of sleep EEG precluding reliable verification of sleep/wake stages. Thus, 29 subjects (nine females) from the early-night group and 26 subjects (12 females) from the late-night group were used for statistical comparisons.

\section{Task}

The task is illustrated in Figure 1A by an example trial. It was the same version of the NRT as described previously by Wagner et al. (2004). On each trial, a different string of eight digits was presented. Each string was composed of the digits 1, 4, and 9. For each string, subjects had to determine a digit defined as the final result (solution) of the task trial. This could be achieved by sequentially processing pairs of digits from left to right according to two simple rules: (1) The "identity rule" states that the result of two identical digits is the same digit (e.g., 1 and 1 gives 1 ) (Fig. $1 \mathrm{~A}$, Response 1). (2) The "difference rule" states that the result of two nonidentical digits is the remaining third digit (e.g., 1 and 4 gives 9) (Fig. 1A, Response 2).

The 1,2, and 3 keys on the PC numeric pad were labeled accordingly 1, 4, and 9 and served as response keys. The entered responses appeared on the screen and remained there until the end of the trial, thereby forming a response sequence below the stimulus sequence. To produce the first response, comparisons are made between the first and the second digits from the stimulus string. After processing the first two digits, comparisons are made between this result (appearing in the line below the stimulus string) and the next digit from the stimulus string, then between the response digit of this new processing and the next stimulus string digit, and so on. Thus, applying the two rules, subjects generate a string of seven responses, with the last one indicating the final result to be confirmed by pressing the "Enter" key on the numeric pad. The time for any single response was limited to $4 \mathrm{sec}$ and to a total of $12 \mathrm{sec}$ for all responses until the Enter key was pressed. Pressing the Enter key was followed by a change of color of the entered final response on the screen, from red to blue. After another 1-sec period, feedback was provided. In case of a correct final result, all digits on the screen, in addition to the final one, changed their color to blue, whereas the German word for "Wrong" appeared on the screen in case of an incorrect final result. The screen was cleared after another $0.5 \mathrm{sec}$, and the next trial started.

Instructions stated that only the final result was to be determined for each trial, and this could be done at any time. Importantly, unmentioned to subjects, all strings were generated according to the same underlying regularity that, if discerned, allowed an early determination of the final result. Specifically, all response sequences had the form $\mathrm{ABCDDCB}$ (with $\mathrm{A}, \mathrm{B}, \mathrm{C}$, and $\mathrm{D}$ representing one of the digits 1,4 , or 9); i.e., the last three responses always mirrored the preceding three responses, so that the second response in each trial coincided with the final result (Fig. 1A, arrows in Response 7). Thus, when gaining insight into this regularity, participants abruptly cut short sequential responding by pressing the Enter key after the second response (R2), whereupon the trial was finished and the next trial started. Note that this regularity is abstract because the actual digit strings and responses changed from trial to trial. Thus, discovery of the rule cannot simply be based on repetition of the same finger movements in all trials.

RTs were measured continuously during task performance, separately for each response in the response string. RT of the first response (R1) was measured as the time from string appearance to the first key press. The RTs of the other responses (R2, R3, R4, R5, R6, R7, Enter) were measured as the time between the previous and the current key press.

\section{Experimental procedure}

The experimental design is presented in Figure 1B. Subjects were tested individually in a sound-attenuated room. As in the previ- 
ous study of Wagner et al. (2004), subjects performed a pre-sleep session of initial practice comprising three task blocks and a postsleep retest session of 10 task blocks, with 30 trials in each block. Insight was automatically identified by the program when at least 24 correct shortcuts within the same block occurred, in which case the task was terminated. Initial practice was preceded by extensive standardized instructions given by the computer, which included a short practice block of 10 task trials. To assure correct understanding of the "identity" and "difference" rule, the practice block was repeated as long as the subject did not perform the 10 trials without mistake. To investigate the effects of different sleep phases, the interval between initial practice and retest was filled with $3 \mathrm{~h}$ of sleep either in the early night, rich in SWS, or in the late night, rich in REM sleep (Fig. 1B).

In the early-night group, subjects reported to the laboratory at $\sim 21: 00 \mathrm{~h}$. After placement of electrodes (for EEG and standard polysomnographic recordings), they performed the three blocks of initial practice (including preceding computer-guided instructions) at $\sim 22: 00 \mathrm{~h}$ and thereafter went to bed at $\sim 23: 00 \mathrm{~h}$. After $3 \mathrm{~h}$ of sleep in the early night they were awakened to perform the 10 blocks of NRT retesting. Subjects in the late-night group reported to the laboratory at $\sim 22: 00 \mathrm{~h}$ and, after placement of electrodes, first slept for $3 \mathrm{~h}$ in the early night (to "consume" SWS) before performing the initial practice at $\sim 2: 30 \mathrm{~h}$. Then, they slept again for another $3 \mathrm{~h}$ in the late night $(\sim 3: 30-6: 30 \mathrm{~h})$, followed by retesting in the morning. In all conditions, sessions also included performance in a short simple choice-response task unrelated to the present study, taking place immediately before and after sleep (i.e., after initial NRT practice and before NRT retesting). They were only awakened from light sleep stages 1 or 2 to avoid cognitive disturbances that can occur after awakenings from SWS or REM sleep. As an additional control, subjective levels of sleepiness, activation, boredom, concentration, and motivation were assessed on five-point scales immediately before and after each session of initial practice and retest.

After the NRT retesting session, subjects filled in a questionnaire to probe their explicit knowledge related to the hidden task structure (beginning with open questions, followed by closed questions), as well as possible strategies used during task performance. An additional behavioral test comprised a speeded task in which 16 different strings were presented and subjects had to indicate the final result to each string within $2 \mathrm{sec}$ after string presentation. This test was performed immediately before administration of the questionnaire.

\section{Assessment of explicit and implicit knowledge of the hidden regularity}

Presence of ExK after sleep was determined from NRT task performance and answers in the post-experimental questionnaire. Questionnaire data confirmed that all subjects whose insight into the hidden structure had been identified automatically by the task program due to the shortcut in sequential responding in the NRT were also able to verbalize the critical explicit rule knowledge correctly in open questions (i.e., using their own words), and they were also able to give correct solutions to new digit strings within 2 sec. Two additional participants were able to exactly verbalize the hidden mirror structure of the response strings in the questionnaire and were therefore also treated as ExK subjects despite their failure to put their explicit knowledge into action. Questionnaire answers also showed that the underlying common structure of all task trials also allowed detecting other shortcut rules than described in Figure 1A. These alternative ways of gaining insight into the hidden structure, likewise always associated with the immediate change to shortcut responding in the NRT, were considered as equivalent ways of acquiring ExK.

ImK was assessed for correct and complete trials on the basis of faster RTs to responses that were predictable due to the mirror structure of the response strings in comparison to unpredictable responses (see Rose et al. 2002, 2005), specifically R6 and R7 in comparison to R3 and R4. Response R5 was not considered, because it was always a direct repetition of R4 and always resulted from application of the simple "identity" rule. Therefore, this response was generally much faster than all other single responses, which could mask RT effects related to implicit learning of the more complex mirror structure (cf. Rose et al. 2002; Lang et al. 2006). Likewise, R2 was not used for analysis because it always resulted from a transition from processing within the stimulus string to processing within stimulus and response strings, producing large variability for $\mathrm{R} 2$ and thus making it inappropriate as a representative undetermined "baseline" response to which determined responses are statistically compared.

For each block and participant, the mean of RTs to R3 and $\mathrm{R} 4$ in each of the 30 single strings were tested against the mean RTs to R6 and R7 by one-way ANOVAs with a repeated-measures factor predictability (unpredictable responses R3/R4 vs. predictable responses R6/R7), accepting the effect of predictability as significant only if $P<0.01$. At the pre-sleep practice session, this analysis was performed for all subjects; at retesting, only for those who did not gain ExK (because these subjects did not perform the full amount of task blocks). A participant was assigned to the pre-sleep ImK subgroup if the $P<0.01$ criterion was met in at least the second or the third block out of the three practice blocks. A participant was assigned to the post-sleep ImK subgroup if the $P<0.01$ criterion was met in at least three consecutive blocks within the ten retest blocks. Subjects who satisfied neither ImK nor ExK criteria were defined as having developed no knowledge about the hidden task regularity (NoK). Analyses on the group level confirmed that ImK subjects, but not NoK subjects, showed substantially faster RTs for predictable responses R3 and R4 compared with unpredictable responses R6 and R7 $(P<0.0001$, for predictability $\times$ NoK/ImK ANOVA interactions for both initial practice and retesting). The specific speeding of predictable relative to unpredictable responses in ImK subjects compared with NoK subjects was also obtained when, despite the objections against including response R2 and, more importantly, response R5 in the evaluation of implicit rule knowledge (see above), all three predictable responses R5-R7 were compared to all three unpredictable responses R2-R4 in the mirror structure $(P<0.001$ and $P=0.015$, for predictability $\times$ NoK/ImK ANOVA interactions at initial practice and retesting, respectively) (see also Fig. 3).

\section{Sleep and statistical analyses}

Sleep was recorded polysomnographically, including EEG recordings from left and right central sites (C3, C4), horizontal and vertical EOG, and EMG from chin electrodes. Sleep stages S1, S2, S3, S4, and REM sleep were classified in 30-sec epochs according to the method of Rechtschaffen and Kales (1968). SWS were calculated as the sum of time spent in sleep stages S3 and S4. Subgroups of NoK and ImK were determined and statistically compared by ANOVAs on RTs as described in the previous section. Sleep parameters were compared by t-tests between early- and late-night sleep and by one-way ANOVA between the subgroups formed according to their knowledge level within the early and late night groups.

To evaluate differences between early and late-night sleep in the development of knowledge about the hidden regularity, $x^{2}$ tests were performed on the distribution of participants assigned to the different subgroups (ExK, ImK, NoK). For subjective ratings of sleepiness, activation, boredom, concentration, and motivation, $2 \times 2 \times 2$ ANOVAs were performed with the between subjects factor night-half (early-night group vs. late-night group) and the within-subjects factors session (initial practice vs. retest) and time within session (beginning vs. end of session).

\section{Acknowledgments}

We thank Vitus Schuknecht for skilled technical assistance. This work was supported by a grant from the Deutsche Forschungsgemeinschaft to R.V. (SFB654/1-1).

\section{References}

Bird, C.M. and Burgess, N. 2008. The hippocampus and memory: Insights from spatial processing. Nat. Rev. Neurosci. 9: 182-194. Born, J., Rasch, B., and Gais, S. 2006. Sleep to remember. Neuroscientist 
12: $410-424$

Brown, R.M. and Robertson, E.M. 2007. Off-line processing: Reciprocal interactions between declarative and procedural memories. $J$. Neurosci. 27: 10468-10475.

Buzsáki, G. 1998. Memory consolidation during sleep: A neurophysiological perspective. J. Sleep Res. (Suppl. 1) 7: 17-23.

Cohen, N.J., Ryan, J., Hunt, C., Romine, L., Wszalek, T., and Nash, C. 1999. Hippocampal system and declarative relational memory: Summarizing the data from functional neuroimaging studies. Hippocampus 9: 83-98.

Dienes, Z., and Perner, J. 1999. A theory of implicit and explicit knowledge. Behav. Brain Sci. 22: 735-755.

Durmer, J.S. and Dinges, D.F. 2005. Neurocognitive consequences of sleep deprivation. Semin. Neurol. 25: 117-129.

Ellenbogen, J.M., Hu, P.T., Payne, J.D., Titone, D., and Walker, M.P. 2007. Human relational memory requires time and sleep. Proc. Natl. Acad. Sci. 104: 7723-7728.

Elsner, B., Hommel, B., Mentschel, C., Drzezga, A., Prinz, W., Conrad, B., and Siebner, H. 2002. Linking actions and their perceivable consequences in the human brain. Neuroimage 17: 364-372.

Fenn, K.M., Nusbaum, H.C., and Margoliash, D. 2003. Consolidation during sleep of perceptual learning of spoken language. Nature 425: 614-616.

Fischer, S., Drosopoulos, S., Tsen, J., and Born, J. 2006. Implicit learning-explicit knowing: a role for sleep in memory system interaction. J. Cogn. Neurosci. 18: 311-319.

Forkstam, C. and Petersson, K.M. 2005. Towards an explicit account of implicit learning. Curr. Opin. Neurol. 18: 435-441.

Fowler, M.J., Sullivan, M.J., and Ekstrand, B.R. 1973. Sleep and memory. Science 179: 302-304.

Frensch, P.A., Haider, H., Rünger, D., Neugebauer, U., Voigt, S., and Werg, J. 2002. In Attention and implicit learning (ed. L. Jimenez), pp. 335-366. John Benjamins, New York.

Gais, S. and Born, J. 2004. Declarative memory consolidation: Mechanisms acting during human sleep. Learn. Mem. 11: 679-685.

Gómez, R.L., Bootzin, R.R., and Nadel, L. 2006. Naps promote abstraction in language-learning infants. Psychol. Sci. 17: 670-674.

Haider, H. and Rose, M. 2007. How to investigate insight: A proposal. Methods 42: 49-57.

Hauptmann, B., Reinhart, E., Brandt, S.A., and Karni, A. 2005. The predictive value of the leveling off of within session performance for procedural memory consolidation. Brain Res. Cogn. Brain Res. 24: $181-189$.

Henke, K., Weber, B., Kneifel, S., Wieser, H.G., and Buck, A. 1999. Human hippocampus associates information in memory. Proc. Natl. Acad. Sci. 96: 5884-5889.

Horne, J.A. 1988. Sleep loss and "divergent" thinking ability. Sleep 11: $528-536$

Kuriyama, K., Stickgold, R., and Walker, M.P. 2004. Sleep-dependent learning and motor-skill complexity. Learn. Mem. 11: 705-713.

Lang, S., Kanngieser, N., Jaskowski, P., Haider, H., Rose, M., and Verleger, R. 2006. Precursors of insight in event-related brain potentials. J. Cogn. Neurosci. 18: 1-15.

Lechner, H.A., Squire, L.R., and Byrne, J.H. 1999. One hundred years of consolidation-remembering Müller and Pilzecker. Learn. Mem. 6: $77-87$.

Maquet, P. 2001. The role of sleep in learning and memory. Science 294: $1048-1052$.

Marshall, L., Helgadóttir, H., Mölle, M., and Born, J. 2007. Boosting slow oscillations during sleep potentiates memory. Nature 444: 610-613.

McClelland, J.L., McNaughton, B.L., and O'Reilly, R.C. 1995. Why there are complementary learning systems in the hippocampus and neocortex: Insights from the successes and failures of connectionist models of learning and memory. Psychol. Rev. 102: 419-457.

McGaugh, J.L. 2000. Memory-a century of consolidation. Science 287: 248-251.

Paller, K.A. and Voss, J.L. 2004. Memory reactivation and consolidation during sleep. Learn. Mem. 11: 664-670.
Peigneux, P., Laureys, S., Fuchs, S., Destrebecqz, A., Collette, F., Delbeuck, X., Phillips, C., Aerts, J., Del Fiore, G., Degueldre, C., et al. 2003. Learned material content and acquisition level modulate cerebral reactivation during post-training rapid-eye-movements sleep. Neuroimage 20: 125-134.

Peigneux, P., Laureys, S., Fuchs, S., Collette, F., Perrin, F., Reggers, J., Phillips, C., Degueldre, C., Del Fiore, G., Aerts, J., et al. 2004. Are spatial memories strengthened in the human hippocampus during slow wave sleep? Neuron 44: 535-545.

Peters, K.R., Smith, V., and Smith, C.T. 2007. Changes in sleep architecture following motor learning depend on initial skill level. $J$. Cogn. Neurosci. 19: 817-829.

Plihal, W. and Born, J. 1997. Effects of early and late nocturnal sleep on declarative and procedural memory. J. Cogn. Neurosci. 9: 534-547.

Plihal, W. and Born, J. 1999. Effects of early and late nocturnal sleep on priming and spatial memory. Psychophysiology 36: 571-582.

Rasch, B., Büchel, C., Gais, S., and Born, J. 2007. Odor cues during slow-wave sleep prompt declarative memory consolidation. Science 315: $1426-1429$.

Reber, A.S. 1989. Implicit learning and tacit knowledge. J. Exp. Psychol. Gen. 118: 219-235.

Reber, P.J. and Squire, L.R. 1994. Parallel brain systems for learning with and without awareness. Learn. Mem. 1: 217-229.

Rechtschaffen, A. and Kales, A.A. 1968. Manual of standardized terminology, techniques and scoring system for sleep stages of human subjects, No. 204. National Institutes of Health, Bethesda, MD.

Robertson, E.M., Pascual-Leone, A., and Press, D.Z. 2004. Awareness modifies the skill-learning benefits of sleep. Curr. Biol. 14: 208-212.

Rose, M., Haider, H., Weiller, C., and Büchel, C. 2002. The role of medial temporal lobe structures in implicit learning: An event-related fMRI study. Neuron 36: 1221-1231.

Rose, M., Haider, H., Weiller, C., and Büchel, C. 2004. The relevance of the nature of learned associations for the differentiation of human memory systems. Learn. Mem. 11: 145-152.

Rose, M., Haider, H., and Büchel, C. 2005. Unconscious detection of implicit expectancies. J. Cogn. Neurosci. 17: 918-927.

Seger, C.A. 1994. Implicit learning. Psychol. Bull. 115: 163-196.

Smith, C. 2001. Sleep states and memory processes in humans: Procedural versus declarative memory systems. Sleep Med. Rev. 5: $491-506$.

Squire, L.R. 1992. Memory and the hippocampus: A synthesis from findings with rats, monkeys, and humans. Psychol. Rev. 99: 195-231.

Wagner, U. and Born, J. 2008. Memory consolidation during sleep: Interactive effects of sleep stages and HPA regulation. Stress 11: $28-41$.

Wagner, U., Gais, S., and Born, J. 2001. Emotional memory formation is enhanced across sleep intervals with high amounts of rapid eye movement sleep. Learn. Mem. 8: 112-119.

Wagner, U., Hallschmid, M., Verleger, R., and Born, J. 2003. Signs of REM sleep dependent enhancement of implicit face memory: A repetition priming study. Biol. Psychol. 62: 197-210.

Wagner, U., Gais, S., Haider, H., Verleger, R., and Born, J. 2004. Sleep inspires insight. Nature 427: 352-355.

Wagner, U., Kashyap, N., Diekelmann, S., and Born, J. 2007. The impact of post-learning sleep vs. wakefulness on recognition memory for faces with different facial expressions. Neurobiol. Learn. Mem. 87: 679-687.

Walker, M.P. and Stickgold, R. 2006. Sleep, memory, and plasticity. Annu. Rev. Psychol. 57: 139-166.

Wilson, M.A. and McNaughton, B.L. 1994. Reactivation of hippocampal ensemble memories during sleep. Science 265: 676-679.

Woltz, D.J., Bell, B.G., Kyllonen, P.C., and Gardner, M.K. 1996. Memory for order of operations in the acquisition and transfer of sequential cognitive skills. J. Exp. Psychol. Learn. Mem. Cogn. 22: 438-457.

Received January 5, 2008; accepted in revised form April 17, 2008. 


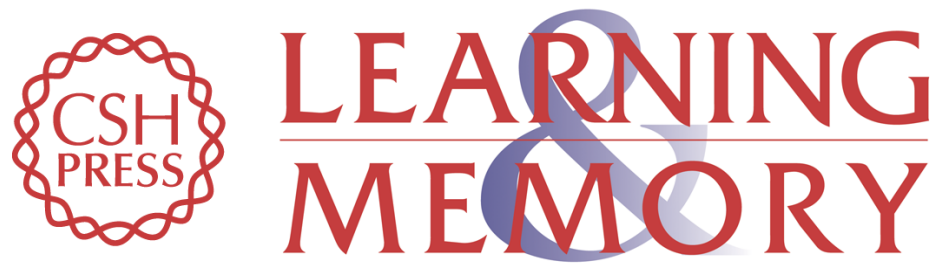

\section{Shifting from implicit to explicit knowledge: Different roles of early- and late-night sleep}

Juliana Yordanova, Vasil Kolev, Rolf Verleger,, et al.

Learn. Mem. 2008, 15:

Access the most recent version at doi:10.1101//m.897908

References This article cites 50 articles, 15 of which can be accessed free at:

http://learnmem.cshlp.org/content/15/7/508.full.html\#ref-list-1

License

Email Alerting Receive free email alerts when new articles cite this article - sign up in the box at the Service top right corner of the article or click here. 